\title{
Groundwater Quality Studies: A Case Study of the Densu Basin, Ghana
}

\author{
C. Tay* and B. Kortatsi \\ CSIR-Water Research Institute, P. O. Box AH 38, Achimota, Accra \\ *Corresponding author; E-mail: collinstay@hotmail.comor korblatay@yahoo.co.uk
}

\begin{abstract}
Groundwater samples from 68 communities within the Densu basin were sampled and analysed over a period of 1 year for various physico-chemical water quality parameters using appropriate certified and acceptable international procedures, in order to assess the water types as well as the suitability of groundwater within the basin for drinking and other domestic uses. The study showed that most of the physico-chemical parameters were within the World Health Organization limits recommended for drinking water. However, a few of the boreholes were slightly acidic. Some boreholes showed high level of mineralization. Borehole $(\mathrm{GaD} 6)$ at Pokuasi recorded the highest conductivity value of $7780.0 \mu \mathrm{S} / \mathrm{cm}$. High levels of nitrates were also recorded in certain communities within the basin. These include Aponsahene (105.8 mg/l), Damang (66.0 mg/l), Adzen Kotoku (61.5 mg/l), Afabeng (50.8 mg/l), New Mangoase (48.3 $\mathrm{mg} / \mathrm{l})$, Asuoatwene $(41.3 \mathrm{mg} / \mathrm{l})$, Potrase $(33.6 \mathrm{mg} / \mathrm{l})$ and Maase $(33.3 \mathrm{mg} / \mathrm{l})$. Correlations between major ions showed expected process-based relationship between $\mathrm{Ca}^{2+}$ and $\mathrm{Cl}^{-}(r=0.86) ; \mathrm{Mg}^{2+}$ and $\mathrm{Cl}^{-}(r=0.84) ; \mathrm{Na}^{+}$and $\mathrm{SO}_{4}^{2-}(r=$ $0.77) ; \mathrm{Na}^{+}$and $\mathrm{Cl}^{-}(r=0.75) ; \mathrm{Mg}^{2+}$ and $\mathrm{SO}_{4}^{2-}(r=0.74) ; \mathrm{Mg}^{2+}$ and $\mathrm{Ca}^{2+}(r=0.71) ; \mathrm{Ca}^{2+}$ and $\mathrm{SO}_{4}^{2-}(r=0.58) ;$ and $\mathrm{K}^{+}$ and $\mathrm{SO}_{4}^{2-}(r=0.51)$, derived mainly from the geochemical and biochemical processes within the aquifer. Two major hydrochemical water types constituting $41 \%$ of groundwater sources within the basin have been delineated. These are $\mathrm{Ca}-\mathrm{Mg}-\mathrm{HCO}_{3}$ water $(19 \%)$ and $\mathrm{Na}-\mathrm{Cl}$ or $\mathrm{Na}-\mathrm{Cl}-\mathrm{HCO}_{3}-\mathrm{Cl}$ water $(22 \%)$ types. Fifty-nine per cent of groundwater sources are mixed waters with no particular cation predominating, and having either $\mathrm{HCO}_{3}^{-}$or $\mathrm{SO}_{4}^{2-}$ ions as the main anion.
\end{abstract}

\section{Introduction}

Groundwater is globally important for human consumption, and changes in quality with subsequent contamination can, undoubtedly, affect human health. It is also important for the support of habitat and for maintaining the quality of baseflow to rivers. The chemical composition of groundwater is a measure of its suitability as a source of water for human consumption and for other purposes, and also influences ecosystem health and function (Appelo \& Postma, 1993). It is, thus, important to detect change and early warnings of change both in natural systems and resulting from pollution. The chemistry (quality) of groundwater reflects input from the atmosphere, soil and water-rock reactions as well as pollutant sources such as mining, land clearance, agriculture, acid precipitation, and domestic and industrial wastes (Appelo \& Postma, 1993).

Groundwater pollution has become a major subject of public concern the world over. Despite the large volume of water that covers the surface of the earth, only $1 \%$ is inland or fresh and easily available for human use (Bockris, 1978). Only 30\% of Ghanaians have access to safe drinking water. Over $50 \%$ of Ghanaians use "unprotected" source of water (Ministry of Health, 1999). Rural communities in Ghana, which form about $70 \%$ of the total population, rely heavily on groundwater as the main source of drinking water. Groundwater quality is, therefore, critical to the quality of health of the people in these areas.

The Densu basin is a coastal river catchment in southern Ghana and a typical rural community like all other rural settlements in Ghana, in which the indigenous population uses groundwater, rivers, streams, ponds and springs as their sources of potable water. The qualities of these water resources vary naturally and widely depending on climate, season, and the geology of the bedrock, as well as anthropogenic activities. The effect of anthropogenic activities (primarily farming) within the Densu basin culminated in a public outcry over the quality of water supplied from the Weija Reservoir (within the basin) which resulted in a ban on farming activities along

West African Journal of Applied Ecology, Vol. 12, 2008 
the Densu river by the Government of Ghana (Daily Graphic, Friday, 14/09/2001), and a call to use water resources judiciously (Daily Graphic, Tuesday, 18/09/2001).

As a result of the growing public concern, the Government signed a Memorandum of Understanding (MOU) in 1999 with the United Nations Centre for Human Settlements-Habitat (UNCHS) to collabo-rate in a project on "Managing Water for African Cities". The primary aim of the project was to find solutions to the increasing water demand in the fast growing African cities, of which Accra and, subsequently, the Densu basin featured.

Natural geochemical and biochemical, as well as anthropogenic impact on ground-water do not only threaten the quality of human health but also poses a threat to sustainable development and management of groundwater resources. Groundwater may, thus, be prone to chemical contamination. It is, therefore, imperative to carry out an extensive groundwater quality assess-ment of the basin, as well as water types of the different water sources, in order to understand the hydrochemistry of ground-water and, therefore, ensure its potable supply in the area. The paper seeks to assess and compare the chemical qualities and water types of groundwater in four districts within the basin to evaluate their suitability for domestic uses.

\section{Materials and methods}

Study area

The Densu basin is located in southern Ghana and lies between latitude $5^{\circ} 30^{\prime} \mathrm{N}$ to $6^{\circ} 20^{\prime} \mathrm{N}$ and longitude $0^{\circ} 10^{\prime} \mathrm{W}$ to $0^{\circ} 35^{\prime} \mathrm{W}$. The basin lies within two distinct climate zones; the dry equatorial climate of the south-eastern coastal plains, and the wet semi-equatorial climate further north from the coast. Both climate zones are characterized by two rainfall regimes with different intensities (Dickson \& Benneh, 1980). The major rainy season extends from May to July and the minor rainfall season occurs between September and November. The mean annual rainfall varies between $800 \mathrm{~mm}$ near the coast to about $1600 \mathrm{~mm}$ in the Densu river source area.

Temperatures are uniformly high throughout the year with a mean annual temperature of about $27{ }^{\circ} \mathrm{C}$; March/April being the hottest $\left(32^{\circ} \mathrm{C}\right)$ while August is the coldest month $\left(23^{\circ} \mathrm{C}\right)$. The upper and middle sections of the basin are covered with semi-deciduous forest with a lush growth of thick and tall trees. In the low-lying areas along the coast, rainfall anomaly gives rise to savanna and scrub forest vegetation with only few isolated trees. There are thickets of bush and grassland, as well as strands and mangrove swamps especially where brackish water prevails.

A complex of precambrian granite and granodiorite with associated gneiss underlie the larger part of the basin (about 90\%). The other formations include rocks of the Upper Birimian that occur at the watershed of the Densu river in the northwest, which consist mainly of metamorphosed lava and tuff, and pyroclastic rocks (Junner, 1940); the rocks of the Togo series, which underlie the south-eastern part of the basin and the area south of Weija to the estuary. The rocks of Togo series were formed from eroded material deposited in the basin. The Togo series consists of sedimentary rocks and their metamorphosed versions (e.g. quartzite, schist, shale and phyllite), which were strongly folded to form what is now called the Akwapim Togo Ranges, extending from the coast near Accra to the Ghana-Togo border. Fig.1 shows the study area and the sampling communities.

West African Journal of Applied Ecology, Vol. 12, 2008 


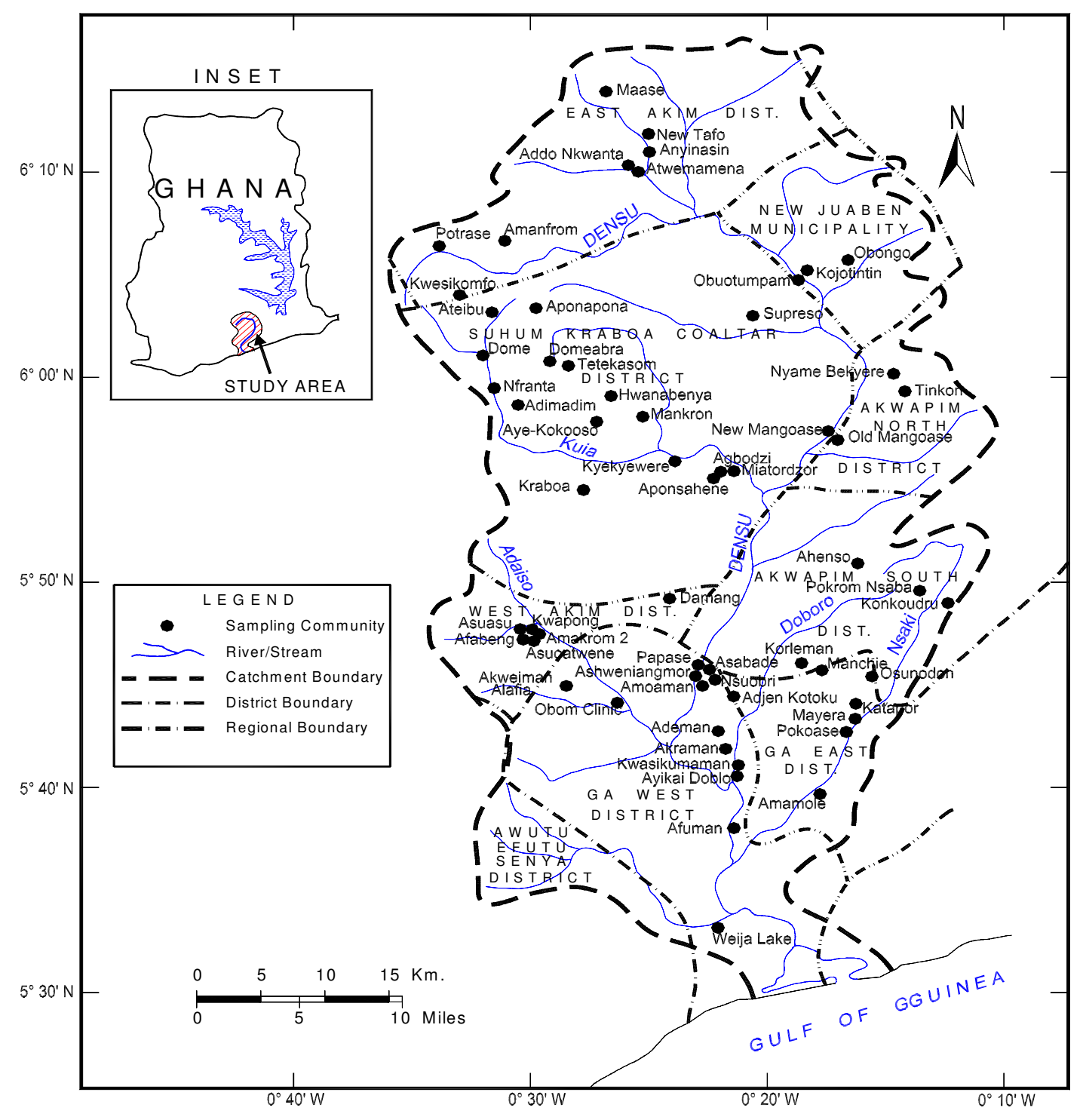

Fig. 1. The Densu basin and the sampling communities

\section{Sampling and analysis}

Sixty-eight water samples were collected from boreholes between February 2005 and December 2005. Sampling protocols described by Claasen (1982) and Barcelona et al. (1985) were strictly observed during sample collection. Samples were collected using 4-1itre acidwashed polypropylene containers. For the determination of heavy metals, each sample was immediately filtered on site through $0.45-\mu \mathrm{m}$ filters on acetate cellulose. Filtrates for metal analysis were transferred into $100-\mathrm{cm}^{3}$ polyethylene bottles and immediately acidified to $p \mathrm{H}<2$ by the addition of Merck ${ }^{\mathrm{TM}}$ ultra pure nitric acid. Samples for anions analyses were collected into $250 \mathrm{~cm}^{3}$ polyethylene bottles without preservation. All samples were stored in an ice-chest and transported to the CSIR-Water Research Institute laboratory, stored in a refrigerator at a temperature of $<4^{\circ} \mathrm{C}$ and analyzed within 1 week. 
Temperature, $p \mathrm{H}$ and electrical conductivity were measured in situ using WTW-Multiline P4 Universal Meter. Chemical analyses of the samples were carried out using appropriate certified and acceptable international procedures outlined in the Standard Methods for the Examination of Water and Wastewater (APHA, 1998). Sodium (Na) and potassium (K) were analysed by flame photometric method, calcium $(\mathrm{Ca})$ by EDTA titration, magnesium $(\mathrm{Mg})$ by calculation after EDTA titration of calcium and total hardness, chloride $(\mathrm{Cl})$ by argentometric titration. Nitratenitrogen was analysed by hydrazine reduction and spectrophotometric determination at $520 \mathrm{~nm}$, and heavy metals were analysed using UNICAM 969 atomic absorption spectro-photometer. An ionic error balance was computed for each chemical sample and used as a basis for checking analytical results. In accordance with international standards, results with ionic balance error greater that $5 \%$ were rejected.

\section{Results and discussion}

The physical parameters of groundwater in the Densu basin are presented in Tables 1a-1d while the chemical parameters are presented in Tables $2 \mathrm{a}-2 \mathrm{~d}$. The water types within the basin are presented in Tables 3a-3d. Groundwater in the various districts of the Densu Basin showed variations in both physical and chemical parameters.

TABLE 1a

Physical parameters of water samples from the Ga District of the Densu basin

$\begin{array}{lllll}\text { Location } & \text { Sample ID } & \mathrm{pH} & \text { Temp. }\left({ }^{\circ} \mathrm{C}\right) & E C(\mu \mathrm{S} / \mathrm{cm}) \\ \text { Amamole } & \mathrm{GaD} 1 & 6.60 & 29.5 & 480.0 \\ \text { Papase } & \mathrm{GaD} 2 & 7.03 & 29.1 & 1089.0 \\ \text { Ademan } & \mathrm{GaD} 3 & 6.18 & 30.6 & 1160.0 \\ \text { Ayikai Doblo } & \mathrm{GaD} 4 & 6.95 & 29.1 & 2211.0 \\ \text { Pokuasi } & \mathrm{GaD} 5 & 6.63 & 27.1 & 4450.0 \\ \text { Pokuasi } & \mathrm{GaD} 6 & 6.71 & 28.7 & 7780.0 \\ \text { Kwasikuma } & \mathrm{GaD} 7 & 6.92 & 29.3 & 2980.0 \\ \text { Papase } & \mathrm{GaD} 8 & 6.41 & 29.2 & 471.0 \\ \text { Akramam } & \mathrm{GaD} 9 & 6.90 & 26.6 & 533.0 \\ \text { Akweiman } & \mathrm{GaD} 10 & 6.77 & 27.8 & 1401.0 \\ \text { Obom Clinic } & \mathrm{GaD} 11 & 6.40 & 28.0 & 964.0 \\ \text { Pokuasi } & \mathrm{GaD} 12 & 6.69 & 26.8 & 3760.0 \\ \text { Mayara Fiase } & \mathrm{GaD} 13 & 6.10 & 28.6 & 1633.0 \\ \text { Katapor } & \mathrm{GaD} 14 & 5.90 & 26.1 & 371.0 \\ \text { Korleman } & \mathrm{GaD} 15 & 5.99 & 26.7 & 644.0 \\ \text { Manchie } & \mathrm{GaD} 16 & 5.87 & 28.9 & 354.0 \\ \text { Amoaman } & \mathrm{GaD} 17 & 6.70 & 30.0 & 1385.0 \\ \text { Nsuobri } & \mathrm{GaD} 18 & 7.17 & 30.2 & 987.0 \\ \text { Ashweniangmor } & \mathrm{GaD} 19 & 6.79 & 27.5 & 1099.0 \\ \text { Asabade } & \mathrm{GaD} 20 & 6.80 & 28.0 & 2310.0 \\ \text { Aden Kotoku } & \mathrm{GaD} 21 & 6.67 & 30.5 & 1160.0 \\ \text { Akramam } & \mathrm{GaD} 22 & 6.70 & 30.0 & 2880.0 \\ \text { Osunodonpe } & \mathrm{GaD} 23 & 6.19 & 28.7 & 809.0 \\ \text { Amamole } & \mathrm{GaD} 24 & 6.73 & 29.0 & 1129.5 \\ \text { Median } & & 6.70 & 28.9 & \end{array}$

West African Journal of Applied Ecology, Vol. 12, 2008 
$\mathrm{GaD}=\mathrm{Ga}$ District

TABLE $1 \mathrm{~b}$

Physical parameters of water samples from the Suhum District of the Densu basin

$\begin{array}{llccc}\text { Location } & \text { Sample ID } & \mathrm{pH} & \text { Temp. }\left({ }^{\circ} \mathrm{C}\right) & E C(\mu \mathrm{S} / \mathrm{cm}) \\ \text { Mankron } & \text { SuD 1 } & 7.40 & 28.3 & 1080.0 \\ \text { Kyekyewere } & \text { SuD 2 } & 6.86 & 28.5 & 1000.0 \\ \text { Kraboa } & \text { SuD 3 } & 6.95 & 27.9 & 478.0 \\ \text { Tetekasom } & \text { SuD 4 } & 6.11 & 27.2 & 134.0 \\ \text { Tetekasom } & \text { SuD 5 } & 6.60 & 26.7 & 328.0 \\ \text { Adimadim } & \text { SuD 6 } & 6.57 & 26.3 & 301.0 \\ \text { Adimadim spring } & \text { SuD 7 } & 6.33 & 25.4 & 217.0 \\ \text { Nfranta } & \text { SuD 8 } & 6.88 & 27.1 & 603.0 \\ \text { Dome 1 } & \text { SuD 9 } & 6.78 & 27.0 & 408.0 \\ \text { Dome 2 } & \text { SuD 10 } & 6.46 & 27.9 & 493.0 \\ \text { Aponapona } & \text { SuD 11 } & 6.63 & 27.0 & 317.0 \\ \text { Ateibu 1 } & \text { SuD 12 } & 6.35 & 27.6 & 417.0 \\ \text { Ateibu 2 } & \text { SuD 13 } & 6.03 & 27.9 & 403.0 \\ \text { Supreso } & \text { SuD 14 } & 5.89 & 27.1 & 267.0 \\ \text { Obuotumpam } & \text { SuD 15 } & 6.80 & 27.3 & 532.0 \\ \text { Obongo } & \text { SuD 16 } & 5.75 & 27.2 & 438.0 \\ \text { Miatordzor } & \text { SuD 17 } & 6.30 & 28.4 & 585.0 \\ \text { Agbodzi } & \text { SuD 18 } & 6.80 & 28.4 & 2340.0 \\ \text { Aponsahene } & \text { SuD 19 } & 6.47 & 29.9 & 1180.0 \\ \text { Aye-Kokooso } & \text { SuD 20 } & 6.73 & 28.3 & 719.0 \\ \text { Hwanabenya } & \text { SuD 21 } & 6.80 & 27.5 & 816.0 \\ \text { Damang } & \text { SuD 22 } & 6.62 & 28.7 & 27.6 \\ \text { Median } & & & 6.61 & 485.5\end{array}$

$\mathrm{SuD}=$ Suhum District

TABLE 1c

Physical parameters of water samples from the Akwapim District of the Densu basin

$\begin{array}{llccc}\text { Location } & \text { Sample ID } & \mathrm{pH} & \text { Temp. }\left({ }^{\circ} \mathrm{C}\right) & \text { EC }(\mu \mathrm{S} / \mathrm{cm}) \\ \text { Kojotintin } & \mathrm{AkD} 1 & 6.11 & 28.0 & 256.0 \\ \text { New Mangoase } & \mathrm{AkD} 2 & 6.36 & 28.2 & 920.0 \\ \text { Old Mangoase } & \mathrm{AkD} \text { 3 } & 6.52 & 28.4 & 683.2 \\ \text { Nyame Bekyere } & \mathrm{AkD} 4 & 6.74 & 28.0 & 628.0 \\ \text { Tinkon } & \mathrm{AkD} \text { 5 } & 6.51 & 29.2 & 443.0 \\ \text { Ahenso } & \mathrm{AkD} 6 & 6.62 & 28.0 & 397.0 \\ \text { Konkourou } & \mathrm{AkD} 7 & 4.49 & 27.1 & 107.0 \\ \text { Median } & & 6.51 & 28.0 & 443.0\end{array}$

$\mathrm{AkD}=$ Akwapim District

West African Journal of Applied Ecology, Vol. 12, 2008 
TABLE 1d

Physical parameters of water samples from the Akim District of the Densu basin

$\begin{array}{lllcc}\text { Location } & \text { Sample ID } & p H & \text { Temp. }\left({ }^{\circ} \mathrm{C}\right) & \text { EC }(\mu \mathrm{S} / \mathrm{cm}) \\ \text { Addo Nkwata 1 } & \text { Ea D1 } & 4.37 & 27.3 & 446.0 \\ \text { Atwemamena } & \text { EaD 2 } & 6.40 & 26.6 & 180.0 \\ \text { Anyinasin } & \text { EaD 3 } & 5.50 & 27.4 & 524.0 \\ \text { Maase 1 } & \text { EaD 4 } & 5.80 & 28.0 & 396.0 \\ \text { Maase 2 } & \text { EaD 5 } & 5.70 & 27.8 & 532.0 \\ \text { Potrase } & \text { EaD 6 } & 6.60 & 26.7 & 612.0 \\ \text { Kwesikomfo } & \text { EaD 7 } & 6.81 & 26.3 & 576.0 \\ \text { Wirenkyem Amanfrom } & \text { EaD 8 } & 6.51 & 27.2 & 653.0 \\ \text { Amakrom } & \text { EaD9 } & 7.08 & 27.7 & 1183.0 \\ \text { Amakrom 2 } & \text { EaD 10 } & 7.20 & 27.1 & 1026.0 \\ \text { Asuoatwene } & \text { EaD 11 } & 6.38 & 28.5 & 922.0 \\ \text { Asuasu } & \text { EaD 12 } & 6.10 & 26.5 & 651.0 \\ \text { Afabeng } & \text { EaD 13 } & 6.60 & 26.5 & 1053.0 \\ \text { Kwapong } & \text { EaD 14 } & 6.55 & 27.4 & 648.0 \\ \text { Ntronso } & \text { EaD15 } & 6.30 & 26.2 & 179.0 \\ \text { Median } & & 6.40 & 27.2 & 612.0\end{array}$

$\mathrm{EaD}=$ East Akim District

TABLE 2a

Major ions, nutrients and trace metal levels of water samples from the Ga District of the Densu basin $\begin{array}{lllllllll}\mathrm{Mg} & \mathrm{Na} & \mathrm{K} & \mathrm{HCO}_{3} & \mathrm{SO}_{4} & \mathrm{Cl} & \mathrm{NO}_{3}\end{array}$ $\mathrm{SiO}_{2}$ $\mathrm{Fe} \quad \mathrm{Mn}$

\begin{tabular}{|c|c|c|c|c|c|c|c|c|c|c|}
\hline Amamole & $\begin{array}{l}24.0 \\
<0.01\end{array}$ & 15.5 & 28.0 & 5.0 & 116.0 & 29.6 & 49.9 & 0.2 & 37.7 & 0.02 \\
\hline Papase & $\begin{array}{c}9.2 \\
<0.01\end{array}$ & 53.9 & 64.0 & 1.5 & 68.0 & 41.1 & 199.0 & 0.4 & 35.9 & 0.05 \\
\hline Ademan & $\begin{array}{c}83.6 \\
0.10\end{array}$ & 73.7 & 27.8 & 7.1 & 250.0 & 26.4 & 199.5 & 20.2 & 15.3 & 0.01 \\
\hline Ayikai Doblo & $\begin{array}{c}68.6 \\
0.50\end{array}$ & 52.4 & 358.0 & 3.6 & 384.0 & 175.0 & 399.5 & 3.3 & 35.6 & 0.38 \\
\hline Pokuasi & $\begin{array}{c}80.2 \\
1.01\end{array}$ & 83.0 & 620.0 & 8.6 & 120.0 & 220.0 & 1226.0 & 0.6 & 19.8 & 0.01 \\
\hline Pokuasi & $\begin{array}{r}537.0 \\
0.41\end{array}$ & 306.0 & 410.0 & 9.2 & 88.0 & 270.0 & 2382.0 & 0.1 & 29.6 & 0.17 \\
\hline Kwasikuma & $\begin{array}{c}64.1 \\
0.05\end{array}$ & 293.7 & 59.0 & 2.8 & 208.0 & 202.0 & 745.0 & 0.6 & 24.2 & 0.01 \\
\hline Papase & $\begin{array}{c}21.5 \\
0.02\end{array}$ & 12.6 & 59.0 & 1.6 & 146.0 & 27.6 & 56.9 & 0.3 & 41.0 & 0.06 \\
\hline Akramam & $\begin{array}{c}41.7 \\
0.21\end{array}$ & 7.7 & 39.0 & 6.0 & 84.0 & 52.4 & 66.7 & 5.8 & 15.3 & 1.76 \\
\hline Akweiman & $\begin{array}{c}30.5 \\
0.01\end{array}$ & 73.8 & 64.0 & 6.9 & 178.0 & 103.0 & 181.0 & 0.7 & 34.2 & 0.01 \\
\hline Obom Clinic & $\begin{array}{c}56.1 \\
0.02\end{array}$ & 37.8 & 81.2 & 2.8 & 154.0 & 98.6 & 204.0 & 0.3 & 39.9 & 0.03 \\
\hline Pokuasi & $\begin{array}{c}56.1 \\
0.05\end{array}$ & 115.0 & 490.0 & 6.5 & 110.0 & 170.0 & 963.0 & 0.7 & 26.6 & 0.07 \\
\hline Mayara Fiase & $\begin{array}{c}96.4 \\
0.01\end{array}$ & 51.1 & 156.6 & 6.7 & 124.0 & 39.5 & 481.4 & 0.2 & 28.8 & 0.17 \\
\hline Katapor & $\begin{array}{c}21.2 \\
0.03\end{array}$ & 12.3 & 27.6 & 3.6 & 36.0 & 11.4 & 81.8 & 0.1 & 9.6 & 0.18 \\
\hline Korleman & $\begin{array}{c}46.5 \\
0.01\end{array}$ & 17.4 & 32.0 & 5.4 & 160.0 & 10.4 & 99.3 & 0.6 & 41.3 & 0.04 \\
\hline
\end{tabular}




\begin{tabular}{|c|c|c|c|c|c|c|c|c|c|c|}
\hline Manchie & $\begin{array}{c}18.2 \\
0.41\end{array}$ & 11.3 & 32.9 & 6.3 & 88.0 & 5.9 & 60.9 & 0.1 & 28.6 & 0.17 \\
\hline Amoaman & $\begin{array}{c}20.9 \\
0.02\end{array}$ & 36.9 & 185.4 & 2.0 & 142.0 & 51.0 & 344.0 & 0.3 & 26.2 & 0.02 \\
\hline Nsuobri & $\begin{array}{c}37.5 \\
0.01\end{array}$ & 16.6 & 88.9 & 1.0 & 74.0 & 25.8 & 208.0 & 0.1 & 8.4 & 0.09 \\
\hline Ashweniangmor & $\begin{array}{c}28.9 \\
0.42\end{array}$ & 74.8 & 55.0 & 7.3 & 196.0 & 86.4 & 184.0 & 0.2 & 27.4 & 0.01 \\
\hline Asabade & $\begin{array}{c}100.0 \\
0.05\end{array}$ & 54.5 & 216.7 & 5.4 & 90.0 & 51.7 & 557.0 & 0.6 & 31.5 & 0.02 \\
\hline Aden Kotoku & $\begin{array}{c}44.0 \\
0.10\end{array}$ & 18.4 & 166.0 & 3.5 & 240.0 & 51.1 & 170.7 & 61.5 & 8.9 & 0.01 \\
\hline Akramam & $\begin{array}{c}68.9 \\
0.02\end{array}$ & 10.1 & 23.3 & 3.8 & 244.0 & 14.8 & 33.7 & 0.4 & 29.8 & 0.01 \\
\hline Osunodonpe & $\begin{array}{l}15.6 \\
<0.01\end{array}$ & 11.2 & 6.1 & 2.6 & 66.0 & 18.0 & 16.9 & 0.2 & 8.4 & 0.90 \\
\hline Amamole & $\begin{array}{c}68.2 \\
0.04\end{array}$ & 24.0 & 98.7 & 4.6 & 248.0 & 108.0 & 101.0 & 0.9 & 35.7 & 0.01 \\
\hline Median & $\begin{array}{c}45.3 \\
0.04\end{array}$ & 37.4 & 64.0 & 4.8 & 133.0 & 51.0 & 191.5 & 0.4 & 28.7 & 0.04 \\
\hline
\end{tabular}

All values in mg/l.

TABLE $2 \mathrm{~b}$

Major ions, nutrients and trace metal levels of water samples from the Suhum District of the Densu basin

\begin{tabular}{|c|c|c|c|c|c|c|c|c|c|c|c|}
\hline & $\mathrm{Ca}$ & $M g$ & $N a$ & $K$ & $\mathrm{HCO}_{3}$ & $\mathrm{SO}_{4}$ & $\mathrm{Cl}$ & $\mathrm{NO}_{3}$ & $\mathrm{SiO}_{2}$ & $F e$ & $M n$ \\
\hline Mankron & 35.2 & 75.4 & 197.3 & 1.7 & 360.0 & 52.7 & 288.0 & 7.8 & 13.0 & 0.01 & 0.02 \\
\hline Kyekyewere & 44.4 & 49.8 & 175.2 & 5.7 & 390.0 & 89.4 & 155.9 & 21.2 & 15.0 & 0.01 & 0.30 \\
\hline Kraboa & 29.0 & 6.9 & 66.5 & 5.7 & 104.0 & 72.9 & 62.2 & 5.6 & 10.0 & 0.01 & 0.30 \\
\hline Tetekasom & 5.9 & 1.9 & 19.5 & 1.5 & 62.0 & 6.0 & 5.6 & 3.6 & 8.0 & 0.01 & 0.01 \\
\hline Tetekasom & 36.8 & 5.6 & 31.5 & 2.2 & 112.0 & 32.9 & 35.6 & 5.5 & 9.0 & 0.10 & 0.30 \\
\hline Adimadim & 23.7 & 10.2 & 26.2 & 2.0 & 86.0 & 37.3 & 32.0 & 0.8 & 10.0 & 0.04 & 0.20 \\
\hline Adimadim spring & 10.4 & 7.2 & 26.8 & 2.0 & 84.0 & 11.7 & 21.0 & 4.2 & 7.5 & 0.40 & 0.01 \\
\hline Nfranta & 59.8 & 10.5 & 48.1 & 1.2 & 156.0 & 66.7 & 58.6 & 6.6 & 12.0 & 0.10 & 0.60 \\
\hline Dome 1 & 46.4 & 13.3 & 20.2 & 6.5 & 144.0 & 46.9 & 26.1 & 4.7 & 11.0 & 0.01 & 0.40 \\
\hline Dome 2 & 34.0 & 13.8 & 19.8 & 2.1 & 126.0 & 12.7 & 60.3 & 0.2 & 10.5 & 0.01 & 0.50 \\
\hline Aponapona & 26.5 & 8.6 & 65.8 & 3.5 & 108.0 & 65.6 & 57.8 & 0.1 & 14.0 & 0.10 & 0.30 \\
\hline Ateibu 1 & 27.8 & 3.8 & 54.7 & 3.4 & 180.0 & 15.4 & 31.5 & 14.3 & 9.3 & 0.02 & 0.10 \\
\hline Ateibu 2 & 12.6 & 9.9 & 24.1 & 2.5 & 99.8 & 5.4 & 28.9 & 2.7 & 11.0 & 0.02 & 0.01 \\
\hline Supreso & 12.9 & 1.4 & 42.1 & 2.4 & 68.1 & 14.7 & 36.1 & 5.3 & 8.9 & 0.01 & 0.02 \\
\hline Obuotumpam & 21.4 & 3.8 & 84.1 & 4.3 & 204.0 & 32.5 & 38.5 & 30.6 & 14.0 & 0.01 & 0.20 \\
\hline Obongo & 34.4 & 13.9 & 56.6 & 4.0 & 88.2 & 15.7 & 119.2 & 12.8 & 15.0 & 0.01 & 0.01 \\
\hline Miatordzor & 36.8 & 13.2 & 118.9 & 3.4 & 220.0 & 48.8 & 103.1 & 11.5 & 15.0 & 0.01 & 0.02 \\
\hline Agbodzi & 117.4 & 132.9 & 304.1 & 6.3 & 372.0 & 281.7 & 702.6 & 18.1 & 11.9 & 0.01 & 0.10 \\
\hline Aponsahene & 66.0 & 17.2 & 219.6 & 3.4 & 400.0 & 87.8 & 142.0 & 105.8 & 13.6 & 0.02 & 0.04 \\
\hline Aye-Kokooso & 30.3 & 17.9 & 195.3 & 3.2 & 450.0 & 35.0 & 90.2 & 27.2 & 15.0 & 0.02 & 0.10 \\
\hline Hwanabenya & 44.3 & 32.8 & 201.0 & 3.1 & 378.0 & 74.3 & 174.7 & 3.0 & 15.0 & 0.02 & 0.10 \\
\hline Damang & 69.2 & 15.7 & 220.8 & 10.5 & 272.0 & 181.3 & 157.5 & 66.0 & 14.0 & 0.01 & 0.10 \\
\hline Median & 34.2 & 11.9 & 61.2 & 3.3 & 150.0 & 42.1 & 59.5 & 6.1 & 12.0 & 0.01 & 0.10 \\
\hline
\end{tabular}

All values in $\mathrm{mg} / \mathrm{l}$

TABLE 2c

Major ions, nutrients and trace metal levels of water samples from the Akwapim District of the Densu basin

West African Journal of Applied Ecology, Vol. 12, 2008 


$\begin{array}{lcrrrrrrrrrr} & \mathrm{Ca} & \mathrm{Mg} & \mathrm{Na} & \mathrm{K} & \mathrm{HCO}_{3} & \mathrm{SO}_{4} & \mathrm{Cl} & \mathrm{NO}_{3} & \mathrm{SiO}_{2} & \mathrm{Fe} & \mathrm{Mn} \\ & & & & & & & & & & & \\ \text { Kojotintin } & 10.8 & 2.7 & 19.3 & 0.6 & 82.0 & 3.6 & 3.4 & 3.2 & 7.0 & 0.04 & 0.01 \\ \text { New Mangoase } & 48.2 & 20.8 & 105.5 & 5.2 & 140.0 & 45.7 & 152.2 & 48.3 & 12.7 & 0.05 & 0.01 \\ \text { Old Mangoase } & 36.5 & 26.7 & 68.6 & 6.0 & 220.0 & 29.2 & 86.9 & 15.9 & 15.0 & 0.04 & 0.02 \\ \text { Nyame Bekyere } & 28.7 & 23.1 & 98.7 & 2.4 & 296.0 & 32.6 & 44.1 & 19.1 & 11.0 & 0.01 & 0.01 \\ \text { Tinkon } & 15.1 & 5.7 & 85.8 & 1.9 & 178.0 & 41.6 & 26.4 & 10.8 & 10.0 & 0.01 & 0.10 \\ \text { Ahenso } & 31.3 & 25.8 & 7.6 & 3.6 & 178.0 & 12.7 & 46.6 & 0.8 & 13.0 & 0.10 & 0.20 \\ \text { Konkoudru } & 3.9 & 2.4 & 10.9 & 3.6 & 12.0 & 8.5 & 20.4 & 5.4 & 8.0 & 0.01 & 0.02 \\ \text { Median } & 28.7 & 20.8 & 68.8 & 3.6 & 178.0 & 29.2 & 44.1 & 10.8 & 11.0 & 0.04 & 0.02\end{array}$

All values in $\mathrm{mg} / \mathrm{l}$

TABLE $2 \mathrm{~d}$

Major ions, nutrients and trace metal levels of water samples from the Akim District of the Densu basin

\begin{tabular}{|c|c|c|c|c|c|c|c|c|c|c|c|c|}
\hline & $\mathrm{Ca}$ & $M g$ & $\mathrm{Na}$ & & $K$ & $\mathrm{HCO}_{3}$ & $\mathrm{SO}_{4}$ & $\mathrm{Cl}$ & $\mathrm{NO}_{3}$ & $\mathrm{SiO}_{2}$ & $\mathrm{Fe}$ & $M n$ \\
\hline Addo Nkwata 1 & 21.2 & 2.9 & 29.3 & 3. & .4 & 9.2 & 16.0 & 65.0 & 26.4 & 13.1 & 0.03 & 0.04 \\
\hline Atwemamena & 18.7 & 6.3 & 8.7 & 1 . & 6 & 91.8 & 1.1 & 10.5 & 0.5 & 15.0 & 0.12 & 0.07 \\
\hline Anyinasin & 32.7 & 2.4 & 37.3 & 5 . & .2 & 65.0 & 10.4 & 91.9 & 0.1 & 8.0 & 0.12 & 0.07 \\
\hline Maase 1 & 23.7 & 1.1 & 13.3 & 3. & .9 & 22.0 & 14.7 & 40.6 & 18.2 & 15.0 & 0.12 & 0.01 \\
\hline Maase 2 & 36.9 & 1.5 & 17.3 & 4. & .3 & 22.0 & 0.0 & 64.3 & 33.3 & 15.0 & 0.01 & 0.32 \\
\hline Potrase & 50.6 & 4.2 & 24.3 & 1. & .2 & 100.0 & 0.1 & 55.3 & 33.6 & 9.0 & 0.06 & 0.35 \\
\hline Kwesikomfo & 29.9 & 13.5 & 22.0 & 3. & .7 & 164.0 & 0.0 & 24.5 & 0.1 & 14.0 & 0.10 & 0.10 \\
\hline Wirenkyem Amanfrom & 43.0 & 28.3 & 53.1 & 1. & .7 & 280.0 & 40.4 & 40.7 & 0.3 & 15.0 & 0.01 & 0.14 \\
\hline Amakrom & 14.5 & 28.2 & 171.3 & 3. & .3 & 35.0 & 114.5 & 60.3 & 10.3 & 15.0 & 0.50 & 0.01 \\
\hline Amakrom 2 & 12.0 & 42.4 & 93.8 & 2 . & .2 & 116.0 & 115.0 & 113.2 & 10.3 & 14.0 & 0.04 & 0.02 \\
\hline Asuoatwene & 21.5 & 20.2 & 122.3 & 3. & .3 & 120.0 & 57.8 & 145.3 & 41.3 & 15.0 & 0.10 & 0.20 \\
\hline Asuasu & 15.1 & 12.2 & 100.6 & 3. & .3 & 130.0 & 57.6 & 95.7 & 4.1 & 13.0 & 0.02 & 0.10 \\
\hline Afabeng & 41.2 & 24.2 & 77.3 & 5 . & .3 & 96.0 & 75.0 & 108.6 & 50.8 & 12.0 & 0.04 & 0.50 \\
\hline Kwapong & 22.9 & 17.7 & 82.2 & 2. & .2 & 190.0 & 60.2 & 71.7 & 4.1 & 15.0 & 0.04 & 0.05 \\
\hline Ntronso & 23.2 & 3.9 & 15.7 & 0 . & .6 & 120.0 & 3.2 & 5.7 & 7.8 & 17.0 & 0.02 & 0.05 \\
\hline Median & 23.2 & 12.2 & 37.3 & 3.3 & 116. & $\begin{array}{ll}0 & 16.0\end{array}$ & 64.3 & 10.3 & 0.04 & 0.07 & & \\
\hline
\end{tabular}

All values in $\mathrm{mg} / \mathrm{l}$

TABLE 3a

Groundwater sources and their water types in the East Akim District

\begin{tabular}{|c|c|c|}
\hline Location & Sample ID & Water Type \\
\hline Addo Nkwata 1 & Ea D1 & $\mathrm{Na}-\mathrm{Ca}-\mathrm{Cl}$ \\
\hline Atwemamena & $\mathrm{EaD} 2$ & $\mathrm{Ca}-\mathrm{Mg}-\mathrm{Na}-\mathrm{HCO}_{3}$ \\
\hline Anyinasin & $\mathrm{EaD} 3$ & $\mathrm{Ca}-\mathrm{Na}-\mathrm{Cl}-\mathrm{HCO}_{3}$ \\
\hline Maase 1 & $\mathrm{EaD} 4$ & $\mathrm{Ca}-\mathrm{Na}-\mathrm{Cl}$ \\
\hline Maase 2 & $\mathrm{EaD} 5$ & $\mathrm{Ca}-\mathrm{Na}-\mathrm{Cl}$ \\
\hline Potrase & $\mathrm{EaD} 6$ & $\mathrm{Ca}-\mathrm{Na}-\mathrm{HCO}_{3}-\mathrm{Cl}$ \\
\hline Kwesikomfo & $\mathrm{EaD} 7$ & $\mathrm{Ca}-\mathrm{Mg}-\mathrm{Na}-\mathrm{HCO}_{3}$ \\
\hline Wirenkyem Amanfrom & $\mathrm{EaD} 8$ & $\mathrm{Mg}-\mathrm{Na}-\mathrm{Ca}-\mathrm{HCO}_{3}$ \\
\hline Amakrom & $\mathrm{EaD} 9$ & $\mathrm{Na}-\mathrm{Mg}-\mathrm{HCO}_{3}-\mathrm{SO}_{4}$ \\
\hline Amakrom 2 & $\mathrm{EaD} 10$ & $\mathrm{Na}-\mathrm{Mg}-\mathrm{Cl}-\mathrm{SO}_{4}-\mathrm{HCO}_{3}$ \\
\hline Asuoatwene & EaD 11 & $\mathrm{Na}-\mathrm{Mg}-\mathrm{Cl}-\mathrm{HCO}_{3}$ \\
\hline Asuasu & $\mathrm{EaD} 12$ & $\mathrm{Na}-\mathrm{Cl}-\mathrm{HCO}_{3}$ \\
\hline
\end{tabular}

West African Journal of Applied Ecology, Vol. 12, 2008 

Afabeng
EaD 13
$\mathrm{Na}-\mathrm{Ca}-\mathrm{Mg}-\mathrm{Cl}-\mathrm{HCO}_{3}-\mathrm{SO}_{4}$
Kwapong
$\mathrm{EaD} 14$
$\mathrm{Na}-\mathrm{Mg}-\mathrm{HCO}_{3}-\mathrm{Cl}$
Ntronso
EaD 15
$\mathrm{Ca}-\mathrm{Na}-\mathrm{HCO}_{3}$
$\mathrm{EaD}=$ East Akim Dsitrict

TABLE $3 \mathrm{~b}$

Groundwater sources and their water types in the Akwapim District

$\begin{array}{lll}\text { Location } & \text { Sample ID } & \text { Water Type } \\ \text { Kojotintin } & \mathrm{AkD} 1 & \mathrm{Na}-\mathrm{Ca}-\mathrm{HCO}_{3} \\ \text { New Mangoase } & \mathrm{AkD} 2 & \mathrm{Na}-\mathrm{Ca}-\mathrm{Mg}-\mathrm{Cl}-\mathrm{HCO}_{3} \\ \text { Old Mangoase } & \mathrm{AkD} 3 & \mathrm{Na}-\mathrm{Mg}-\mathrm{Ca}-\mathrm{HCO}_{3}-\mathrm{Cl} \\ \text { Nyame Bekyere } & \mathrm{AkD} 4 & \mathrm{Na}-\mathrm{Mg}-\mathrm{HCO} \\ \text { Tinkon } & \mathrm{AkD} 5 & \mathrm{Na}-\mathrm{HCO} \\ \text { Ahenso } & \mathrm{AkD} 6 & \mathrm{Mg}-\mathrm{Ca}_{3} \mathrm{HCO}_{3}-\mathrm{Cl} \\ \text { Konkourou } & \mathrm{AkD} 7 & \mathrm{Na}-\mathrm{Cl}\end{array}$

$\mathrm{AkD}=$ Akwapim District

TABLE 3c

Groundwater sources and their water types in the Ga District

\begin{tabular}{|c|c|c|}
\hline Location & Sample ID & Water Type \\
\hline Amamole & $\mathrm{GaD} 1$ & $\mathrm{Mg}-\mathrm{Na}-\mathrm{Ca}-\mathrm{HCO}_{3}-\mathrm{Cl}$ \\
\hline Papase & $\mathrm{GaD} 2$ & $\mathrm{Mg}-\mathrm{Na}-\mathrm{Cl}$ \\
\hline Ademan & $\mathrm{GaD} 3$ & $\mathrm{Mg}-\mathrm{Ca}-\mathrm{Cl}-\mathrm{HCO}_{3}$ \\
\hline Ayikai Doblo & $\mathrm{GaD} 4$ & $\mathrm{Na}-\mathrm{Cl}-\mathrm{HCO}_{3}$ \\
\hline Pokuasi & $\mathrm{GaD} 5$ & $\mathrm{Na}-\mathrm{Cl}$ \\
\hline Pokuasi & $\mathrm{GaD} 6$ & $\mathrm{Ca}-\mathrm{Mg}-\mathrm{Na}-\mathrm{Cl}$ \\
\hline Kwasikuma & $\mathrm{GaD} 7$ & $\mathrm{Mg}-\mathrm{Cl}$ \\
\hline Papase & $\mathrm{GaD} 8$ & $\mathrm{Na}-\mathrm{Ca}-\mathrm{Mg}-\mathrm{HCO}_{3}-\mathrm{Cl}$ \\
\hline Akramam & $\mathrm{GaD} 9$ & $\mathrm{Ca}-\mathrm{Na}-\mathrm{Cl}-\mathrm{HCO}_{3}-\mathrm{SO}_{4}$ \\
\hline Akweiman & $\mathrm{GaD} 10$ & $\mathrm{Mg}-\mathrm{Na}-\mathrm{Cl}-\mathrm{HCO}_{3}-\mathrm{SO}_{4}$ \\
\hline Obom Clinic & $\mathrm{GaD} 11$ & $\mathrm{Na}-\mathrm{Mg}-\mathrm{Ca}-\mathrm{Cl}-\mathrm{HCO}_{3}-\mathrm{SO}_{4}$ \\
\hline Pokuasi & $\mathrm{GaD} 12$ & $\mathrm{Na}-\mathrm{Mg}-\mathrm{Cl}$ \\
\hline Mayara Fiase & $\mathrm{GaD} 13$ & $\mathrm{Na}-\mathrm{Ca}-\mathrm{Mg}-\mathrm{Cl}$ \\
\hline Katapor & $\mathrm{GaD} 14$ & $\mathrm{Na}-\mathrm{Ca}-\mathrm{Mg}-\mathrm{Cl}$ \\
\hline Korleman & $\mathrm{GaD} 15$ & $\mathrm{Ca}-\mathrm{Mg}-\mathrm{Na}-\mathrm{Cl}-\mathrm{HCO}_{3}$ \\
\hline Manchie & $\mathrm{GaD} 16$ & $\mathrm{Na}-\mathrm{Mg}-\mathrm{Ca}-\mathrm{Cl}-\mathrm{HCO}_{3}$ \\
\hline Amoaman & $\mathrm{GaD} 17$ & $\mathrm{Na}-\mathrm{Mg}-\mathrm{Cl}$ \\
\hline Nsuobri & $\mathrm{GaD} 18$ & $\mathrm{Na}-\mathrm{Ca}-\mathrm{Cl}$ \\
\hline Ashweniangmor & $\mathrm{GaD} 19$ & $\mathrm{Mg}-\mathrm{Na}-\mathrm{Cl}-\mathrm{HCO}_{3}$ \\
\hline Asabade & $\mathrm{GaD} 20$ & $\mathrm{Na}-\mathrm{Ca}-\mathrm{Mg}-\mathrm{Cl}$ \\
\hline Adzen Kotoku & $\mathrm{GaD} 21$ & $\mathrm{Na}-\mathrm{Ca}-\mathrm{Cl}-\mathrm{HCO}_{3}$ \\
\hline Akramam & $\mathrm{GaD} 22$ & $\mathrm{Ca}-\mathrm{HCO}_{3}$ \\
\hline Osunodonpe & $\mathrm{GaD} 23$ & $\mathrm{Mg}-\mathrm{Ca}-\mathrm{HCO}_{3}-\mathrm{Cl}$ \\
\hline Amamole & $\mathrm{GaD} 24$ & $\mathrm{Na}-\mathrm{Ca}-\mathrm{Mg}-\mathrm{HCO}_{3}-\mathrm{Cl}-\mathrm{SO}_{4}$ \\
\hline
\end{tabular}


$\mathrm{GaD}=\mathrm{Ga}$ District

TABLE 3d

Groundwater sources and their water types in the Suhum District.

\begin{tabular}{|c|c|c|}
\hline Location & Sample ID & Water Type \\
\hline Mankron & SuD 1 & $\mathrm{Na}-\mathrm{Mg}-\mathrm{Cl}-\mathrm{HCO}_{3}$ \\
\hline Kyekyewere & $\mathrm{SuD} 2$ & $\mathrm{Na}-\mathrm{Mg}-\mathrm{HCO}_{3}-\mathrm{Cl}$ \\
\hline Kraboa & SuD 3 & $\mathrm{Na}-\mathrm{Ca}-\mathrm{Cl}-\mathrm{HCO}_{3}-\mathrm{SO}_{4}$ \\
\hline Tetekasom & SuD 4 & $\mathrm{Na}-\mathrm{Ca}-\mathrm{HCO}_{3}$ \\
\hline Domeabra & SuD 5 & $\mathrm{Ca}-\mathrm{Na}-\mathrm{HCO}_{3}-\mathrm{Cl}$ \\
\hline Adimadim & SuD 6 & $\mathrm{Ca}-\mathrm{Na}-\mathrm{Mg}-\mathrm{HCO}_{3}-\mathrm{Cl}-\mathrm{SO}_{4}$ \\
\hline Adimadim spring & SuD 7 & $\mathrm{Na}-\mathrm{Mg}-\mathrm{Ca}-\mathrm{HCO}_{3}-\mathrm{Cl}$ \\
\hline Nfranta & SuD 8 & $\mathrm{Ca}-\mathrm{Na}-\mathrm{HCO}_{3}-\mathrm{Cl}-\mathrm{SO}_{4}$ \\
\hline Dome 1 & SuD 9 & $\mathrm{Ca}-\mathrm{Mg}-\mathrm{Na}-\mathrm{HCO}_{3}-\mathrm{SO}_{4}$ \\
\hline Dome 2 & SuD 10 & $\mathrm{Ca}-\mathrm{Mg}-\mathrm{Na}-\mathrm{HCO}_{3}-\mathrm{Cl}$ \\
\hline Aponapona & SuD 11 & $\mathrm{Na}-\mathrm{Ca}-\mathrm{HCO}_{3}-\mathrm{Cl}-\mathrm{SO}_{4}$ \\
\hline Ateibu 1 & SuD 12 & $\mathrm{Na}-\mathrm{Ca}-\mathrm{HCO}_{3}-\mathrm{Cl}$ \\
\hline Ateibu 2 & SuD 13 & $\mathrm{Na}-\mathrm{Mg}-\mathrm{Ca}-\mathrm{HCO}_{3}-\mathrm{Cl}$ \\
\hline Supreso & SuD 14 & $\mathrm{Na}-\mathrm{Ca}-\mathrm{HCO}_{3}-\mathrm{Cl}$ \\
\hline Obuotumpam & SuD 15 & $\mathrm{Na}-\mathrm{Ca}-\mathrm{HCO}_{3}-\mathrm{Cl}$ \\
\hline Obongo & SuD 16 & $\mathrm{Na}-\mathrm{Ca}-\mathrm{Mg}-\mathrm{Cl}-\mathrm{HCO}_{3}$ \\
\hline Miatordzor & SuD 17 & $\mathrm{Na}-\mathrm{Ca}-\mathrm{HCO}_{3}-\mathrm{Cl}$ \\
\hline Agbodzi & SuD 18 & $\mathrm{Na}-\mathrm{Mg}-\mathrm{Cl}$ \\
\hline Aponsahene & SuD 19 & $\mathrm{Na}-\mathrm{Ca}-\mathrm{HCO}_{3}-\mathrm{Cl}$ \\
\hline Aye-Kokooso & SuD 20 & $\mathrm{Na}-\mathrm{HCO}_{3}-\mathrm{Cl}$ \\
\hline Hwanabenya & SuD 21 & $\mathrm{Na}-\mathrm{Mg}-\mathrm{HCO}_{3}-\mathrm{Cl}$ \\
\hline Damang & SuD 22 & $\mathrm{Na}-\mathrm{Ca}-\mathrm{HCO}_{3}-\mathrm{Cl}-\mathrm{SO}_{4}$ \\
\hline
\end{tabular}

\section{Physical characteristics}

The median values of the water temperature ranged from $27.2{ }^{\circ} \mathrm{C}$ in the Akim District to 28.9 ${ }^{\circ} \mathrm{C}$ in the $\mathrm{Ga}$ District. These values are within the natural background levels of $22-29{ }^{\circ} \mathrm{C}$ for waters in the tropics (Stumm \& Morgan, 1981). The water $p \mathrm{H}$ varied slightly, with median values ranging from 6.40 in the Akim District to 6.7 in the Ga District. The $p \mathrm{H}$ levels of groundwater in the Densu basin are within the natural background levels of 6.5-9.0 (Stumm \& Morgan, 1981) although Korleman (5.99), Katapor (5.9), Manchie (5.87) in the Ga District (Table 1a), Supreso (5.89) and Obongo (5.75) in the Suhum District (Table 1b), Konkourou (4.49) in the Akwapim District (Table 1c) and Addo Nkwanta (4.37), Anyinasin (5.50), Maase (5.7 and 5.80) in the Akim District (Table 1d) had $p \mathrm{H}$ levels outside the natural background levels. Groundwater acidification in these areas could originate from different anthropogenic processes such as excessive use of ammonia and manure as fertilizers in farming activities.

The oxidation of ammonia by oxygen can be described by the following reaction equation:

$$
\mathrm{NH}_{4}^{+}+2 \mathrm{O}_{2}=\mathrm{NO}_{3}^{-}+2 \mathrm{H}^{+}+\mathrm{H}_{2} \mathrm{O} \ldots(1)
$$


This reaction is the main acidifying process in the soil. When plants consume all the nitrate $\mathrm{NO}_{3}^{-}$ ) produced in equation 1, the proton production due to the oxidation process is balanced by the $\mathrm{HCO}_{3}^{-}$produc-tion of the denitrification process as presented in equation 2.

$$
5 \mathrm{CH}_{2} \mathrm{O}+4 \mathrm{NO}_{3}^{-}=2 \mathrm{~N}_{2}+4 \mathrm{HCO}_{3}^{-}+\mathrm{CO}_{2}+3 \mathrm{H}_{3} \mathrm{O} \ldots
$$

However, the ubiquitous presence of nitrate in aquifers is ample proof of the opposite, and nitrification of ammonia must be considered as an important acidifying process. The net effect of nitrification on the $p \mathrm{H}$ of recharge water is largely determined by the amount of lime, which is applied together with fertilizers to the soil.

Oxidation of pyrite $\left(\mathrm{FeS}_{2}\right)$. Pyrite is found at least in small quantities in most reducing sediments and a lowering of the groundwater table, caused by production wells, may result in the oxidation of pyrite. This reaction can be described by the following overall reaction:

$$
2 \mathrm{FeS}_{2}+15 / 2 \mathrm{O}_{2}+5 \mathrm{H}_{2} \mathrm{O} \rightarrow 2 \mathrm{FeOOH}+4 \mathrm{SO}_{4}{ }^{2-}+8 \mathrm{H}^{+}
$$

This reaction is one of the most strongly acid-producing reactions in nature. Thus, anthropogenic processes have significant effect on the acidification of groundwater. The box plots (Fig. 2) show that ground-water in the Ga District recorded the highest median $p \mathrm{H}$, while the Akim District recorded the lowest median $p \mathrm{H}$.

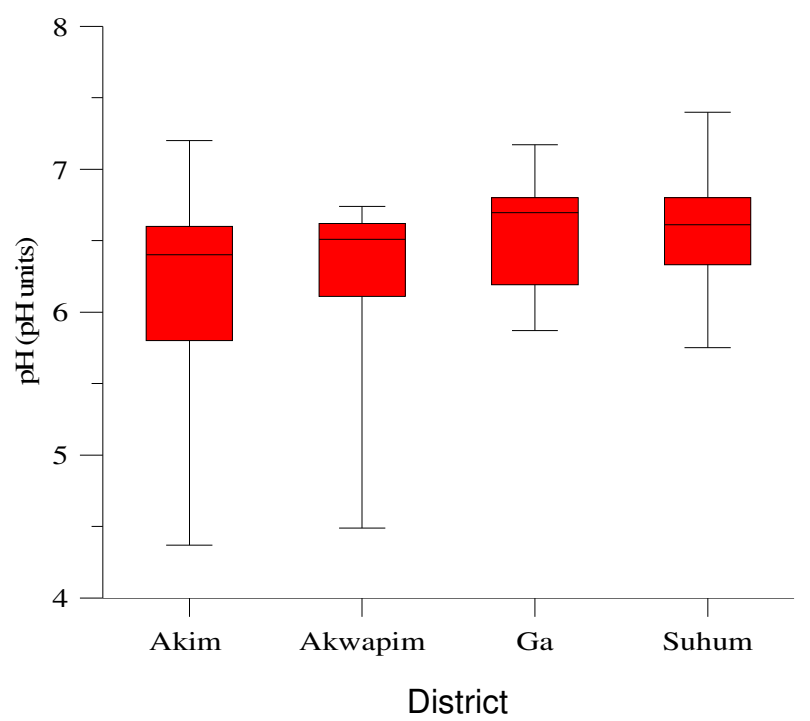

Fig. 2. $p \mathrm{H}$ levels in the groundwater of the Densu basin.

The median conductivity levels varied between $443.0 \mu \mathrm{S} / \mathrm{cm}$ in the Akwapim District and $1129.5 \mu \mathrm{S} / \mathrm{cm}$ in the Ga District. Generally, groundwater in the Densu basin recorded relatively low conductivity values, except for Ayikai Doblo (2211.0 $\mu \mathrm{S} / \mathrm{cm})$, Pokuasi (3760.0, 4450.0 and $7780.0 \mu \mathrm{S} / \mathrm{cm})$, Kwasikuma $(2980.0 \mu \mathrm{S} / \mathrm{cm})$, Mayara Fiase $(1633.0 \mu \mathrm{S} / \mathrm{cm})$, Asabade $(2310.0$ $\mu \mathrm{S} / \mathrm{cm})$ and Akramam $(2880.0 \mu \mathrm{S} / \mathrm{cm})$ in the Ga District, and Agbodzi $(2340.0 \mu \mathrm{S} / \mathrm{cm})$ in the Suhum District. This is an indication of the extent of mineralization, which is dependent on $p \mathrm{H}$, and, therefore, suggestive of heavy impact of human or natural geochemical and biochemical activities in these areas. As can be seen in the box plots (Fig. 3), the Ga District recorded elevated conductivity values, with Pokuasi $(7780.0 \mu \mathrm{S} / \mathrm{cm}$ ) recording the highest (Table 1a).

West African Journal of Applied Ecology, Vol. 12, 2008 


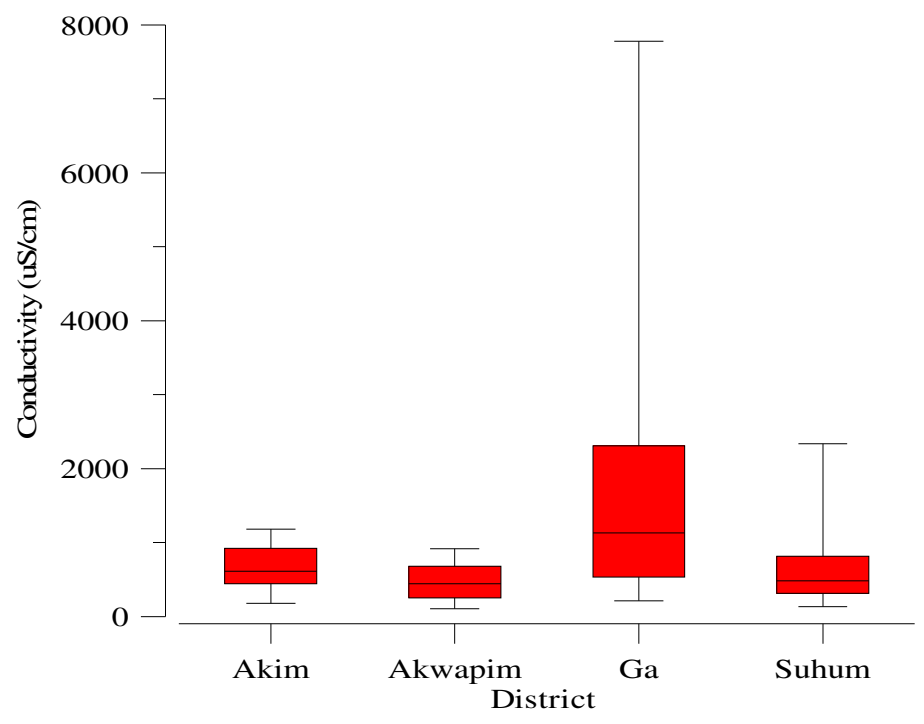

Fig. 3. Conductivity levels in the groundwater of the Densu basin.

\section{Chemical characteristics}

Major ions. Groundwater in the Densu basin is characterized by low chloride levels with median values ranging from $44.1 \mathrm{mg} / \mathrm{l}$ in the Akwapim District to $191.5 \mathrm{mg} / \mathrm{l}$ in the Ga District. These values are within the acceptable WHO limits of $250 \mathrm{mg} / \mathrm{l}$ for drinking water. However, a few communities recorded elevated chloride values. These include Mankrong $(288.0 \mathrm{mg} / \mathrm{l})$ and Agbodzi (702.6 mg/l) in the Suhum District, and Ayikai Doblo (399.5 mg/l), Pokuasi ( 963 $\mathrm{mg} / \mathrm{l}, 1226.0 \mathrm{mg} / \mathrm{l}$ and $2382 \mathrm{mg} / \mathrm{l})$, Kwasikuma (745 mg/l), Mayira Fiase (481.4 mg/l), Amoaman (344.0 mg/l) and Asabade ( $557.0 \mathrm{mg} / \mathrm{l})$ in the Ga District.

According to the classification of Dallas \& Day (1993), chloride ions are non-cumulative toxins; however, excessive amounts of it, if taken over a long period of time, can constitute a health hazard. As can be seen in the box plots (Fig. 4), the Ga District recorded relatively higher chloride levels compared to the other districts. This could originate from natural sources such as the intrusion of sea-water, because of the nearness of the Ga District to the coast. It may also be due to anthropogenic activities such as the intrusion of sewage effluent. The high chloride values in the waters of the Ga District may increase the corrosive ability of the waters in these areas, particularly in low alkalinity waters. 


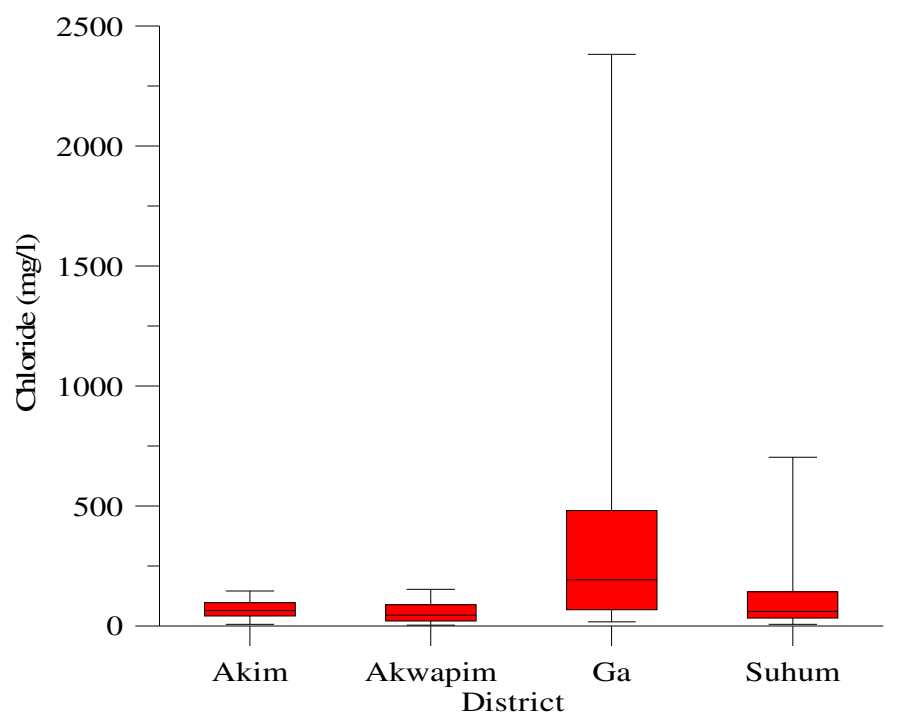

Fig. 4. Chloride levels in the groundwater of the Densu basin.

Nutrients. Low to medium nitrate levels characterize groundwater in the Densu basin with median values ranging from $0.4 \mathrm{mg} / \mathrm{l}$ in the Ga District to $10.8 \mathrm{mg} / \mathrm{l}$ in the Akwapim District. Aponsahene $(105.8 \mathrm{mg} / \mathrm{l})$ recorded the highest nitrate concentration in the basin. The high nitrate values in the waters of the basin could originate from the use of ammonia and manure as fertilizers in farming activities and/or human and animal wastes. The amounts of nitrate, nitrite and ammonia present in natural waters in the form of nitrogen are of great interest because of their nutrient levels. $\mathrm{NH}_{3}-\mathrm{N}, \mathrm{NO}_{3}-\mathrm{N}$ and $\mathrm{NO}_{2}-\mathrm{N}$ are consi-dered to be non-cumulative toxins (Dallas \& Day, 1993). When present in high concentrations, $\mathrm{NO}_{3}-\mathrm{N}$ may give rise to potential health risks particularly in pregnant women and bottle-fed infants (Kempster et al., 1997; Kelter et al., 1997; Cotruvo, 1988; Bush \& Mayer, 1982). At elevated concentrations, $\mathrm{NO}_{3}-\mathrm{N}$ is also known to result in cyanosis in infants (Comly, 1945). Above $10 \mathrm{mg} / \mathrm{l}$, appreciable amounts of $\mathrm{NO}_{3}-\mathrm{N}$ may be produced from $\mathrm{NH}_{3}-\mathrm{N}$ under suitable anaerobic conditions (WHO, 1993; Kempster et al., 1997).

The nitrate levels in the groundwater at Ademan $(20.2 \mathrm{mg} / \mathrm{l})$, Adzen Kotoku $(61.5 \mathrm{mg} / \mathrm{l})$ in the Ga District; Kyekyewere (21.2 mg/l), Ateibu (34.3 mg/l), Obuotumpan (30.6 mg/l, Agbodzi (18.1 $\mathrm{mg} / \mathrm{l})$, Aponsahene $(105.8 \mathrm{mg} / \mathrm{l})$, Aye Kokoosu $(27.2 \mathrm{mg} / \mathrm{l})$, and Damang $(66.0 \mathrm{mg} / \mathrm{l})$ in the Suhum District; New Mangoase (48.3 mg/l), Old Mangoase $(15.9 \mathrm{mg} / \mathrm{l})$, and Nyame Bekyere $(19.9 \mathrm{mg} / \mathrm{l})$, in the Akwapim District; Addo Nkwanta $(26.4 \mathrm{mg} / \mathrm{l} \mathrm{Maase}(18.2$ and $33.3 \mathrm{mg} / \mathrm{l})$, Potrase $(33.6 \mathrm{mg} / \mathrm{l})$, Asuoatwene $(41.3 \mathrm{mg} / \mathrm{l})$, and Afabeng $(50.8 \mathrm{mg} / \mathrm{l})$ in the Akwapim District are at such levels capable of causing methemoglobin 'blue baby syndrome' in infants and a suspect for stomach cancer in adults (Baird, 1995). As can be seen in the box plots (Fig. 5), the Akim District recorded the highest median nitrate level. The levels of nitrates in these areas may be attributed to extensive use of ammonia and manure in farming activities. 


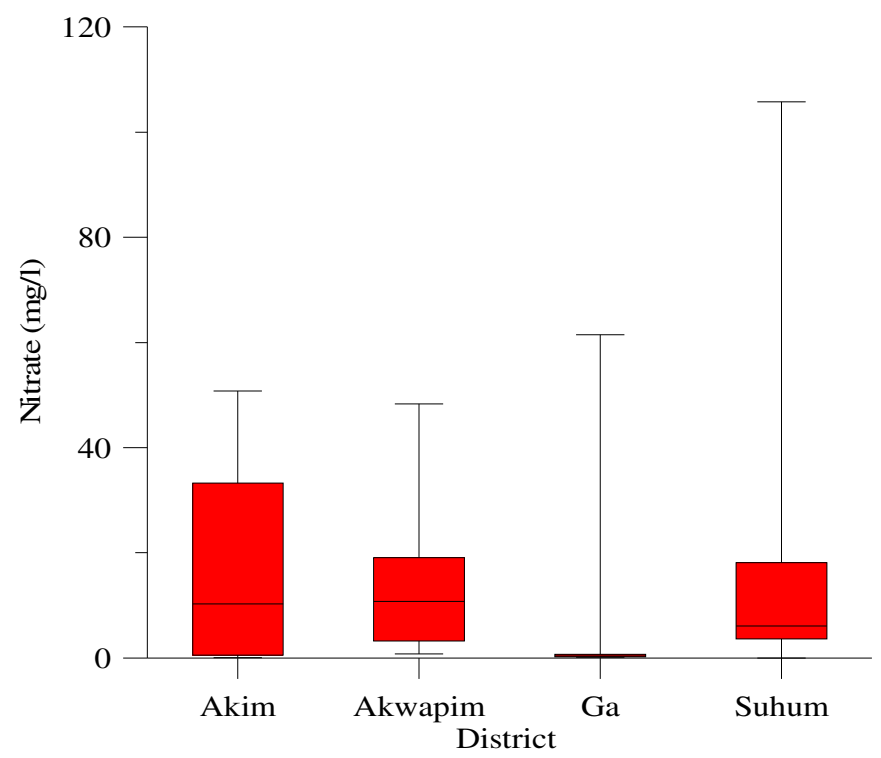

Fig.5. Nitrate levels in the groundwater of the Densu basin

Heavy metals. Heavy metal (iron and manganese) levels in the groundwater of the basin are relatively low. Nevertheless, significant iron $(\mathrm{Fe})$ levels were recorded at Akraman $(1.76 \mathrm{mg} / \mathrm{l})$ and Osunodompe $(0.9 \mathrm{mg} / \mathrm{l})$ in the Ga District, and Amakrom $(0.5 \mathrm{mg} / \mathrm{l})$ in the Akim District, which were all above the WHO limit of $0.3 \mathrm{mg} / \mathrm{l}$ for drinking water. The high Fe levels in these waters could be attributed to natural sources such as the geochemical and biochemical processes within their aquifers. Fig. 6 presents the box plots of Fe in the basin. As shown in Fig. 6, the Akim District recorded the highest median Fe value.

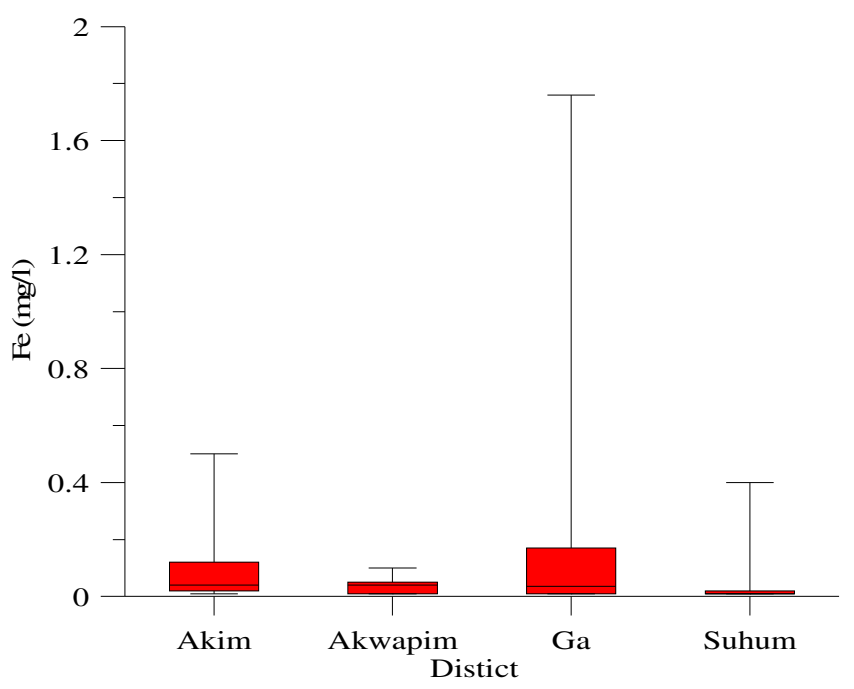

Fig. 6. Fe levels inthe groundwater of the Densu basin

Manganese (Mn) is one of the more biogeochemical and active transition metals in aquatic environment (Evans et al., 1977) and often occurs with iron (Fe). Its concentrations in some communities were also significantly above the WHO limit of $0.1 \mathrm{mg} / \mathrm{l}$ for drinking water. These communities included Pokuasi (0.41 and $1.01 \mathrm{mg} / \mathrm{l})$, Ayikai Doblo (0.5 mg/l), Akraman (0.21 
$\mathrm{mg} / \mathrm{l})$, Manchie $(0.4 \mathrm{mg} / \mathrm{l})$, Ashwenangmor $(0.42 \mathrm{mg} / \mathrm{l})$ in the Ga District; Kyekyewere $(0.3 \mathrm{mg} / \mathrm{l})$, Krabon $(0.3 \mathrm{mg} / \mathrm{l})$, Tetekasom $(0.3 \mathrm{mg} / \mathrm{l})$, Adimadin $(0.2 \mathrm{mg} / \mathrm{l})$, Nfranta $(0.6 \mathrm{mg} / \mathrm{l})$, Dome $(0.4$ and $0.5 \mathrm{mg} / \mathrm{l})$ and Aponapona $(0.3 \mathrm{mg} / \mathrm{l})$ in the Suhum District; Ahenso $(0.2 \mathrm{mg} / \mathrm{l})$ in the Akwapim District and Maase $(0.32 \mathrm{mg} / \mathrm{l})$, Potrase $(0.35 \mathrm{mg} / \mathrm{l})$, Asuoa-twene $(0.2 \mathrm{mg} / \mathrm{l})$ and Afaben $(0.5 \mathrm{mg} / \mathrm{l})$ in the Akim District.

Fig. 7 presents the box plots of Mn in the basin. As shown in Fig 7, the Suhum District recorded the highest median Mn value. Though the presence of high Fe and $\mathrm{Mn}$ concentrations in water does not pose human health problems, these concen-trations have the ability of discoloring water, i.e. high $\mathrm{Fe}$ concentrations results in reddish brown coloration, while high $\mathrm{Mn}$ concentrations results in black coloration, which both result to the staining of laundry and sanitary wares (WHO, 1996).

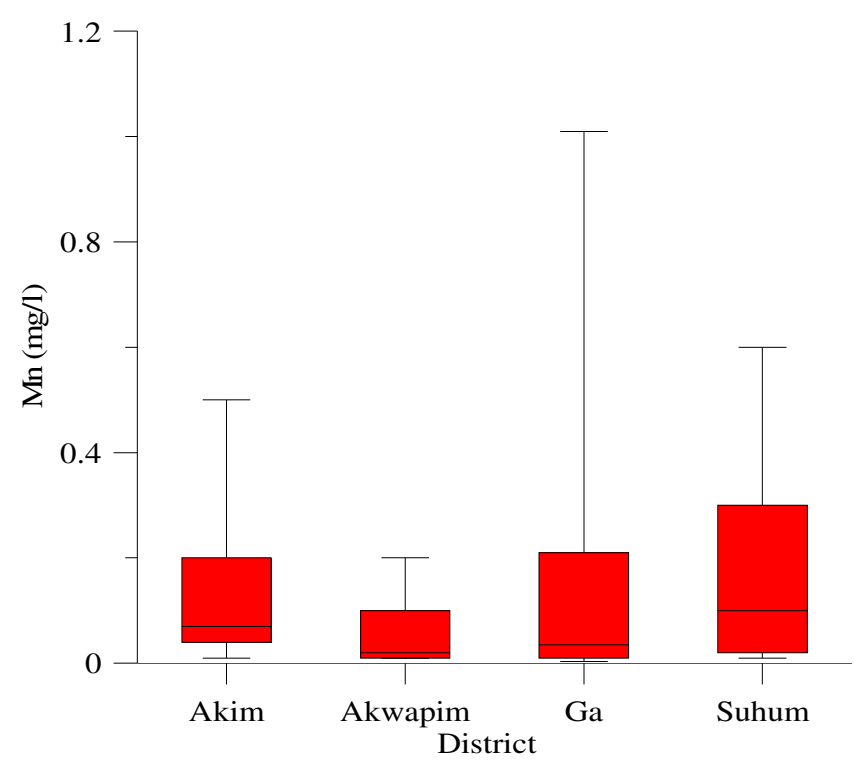

Fig. 7. Mn levels in the groundwater of the Densu basin

Correlations among chemical parameters are presented in Table 3 . The positively strong correlation between $\mathrm{Ca}^{2+}$ and $\mathrm{Cl}^{-}(r=0.86) ; \mathrm{Mg}^{2+}$ and $\mathrm{Cl}^{-}(r=0.84) ; \mathrm{Na}^{+}$and $\mathrm{SO}_{4}^{2-}(r=0.77)$; $\mathrm{Na}^{+}$and $\mathrm{Cl}^{-}(r=0.75) ; \mathrm{Mg}^{2+}$ and $\mathrm{SO}_{4}^{2-}(r=0.74) ; \mathrm{Mg}^{2+}$ and $\mathrm{Ca}^{2+}(r=0.71)$; and a weak positive correlation between $\mathrm{Ca}^{2+}$ and $\mathrm{SO}_{4}^{2-}(r=0.58)$; and $\mathrm{K}^{+}$and $\mathrm{SO}_{4}^{2-}(r=0.51)$, derived mainly from the geochemical and biochemical processes within the aquifer, suggest that these pair of ions have a common origin. Addi-tionally, the strong correla-tion between conductivity (EC) and $\mathrm{Cl}^{-}(r=$ $0.95), \mathrm{Ca}^{2+}(r=0.83), \mathrm{Mg}^{2+}(r=0.82), \mathrm{Na}^{+}(r=0.76)$, and $\mathrm{SO}_{4}^{2-}(r=0.76)$, is suggestive of the contribution of these ions to the salinity of groundwater in the Densu basin.

Water samples. Fig. 8 shows Piper Trilinear Plot (Piper, 1994) of major ions concentrations of water samples in the Densu basin. Two major hydrochemical water types have been delineated. These are $\mathrm{Ca}-\mathrm{Mg}-\mathrm{HCO}_{3}$ water where the chemical properties of the water are dominated by alkaline earths and weak acids (Karanth, 1987). This water type occupies the section of the diamond shape designated as II and represents $19 \%$ of groundwater in the Densu basin. This percentage is made up of 7\%, 3\%, 3\%, and 6\% contributions from the East Akim, Akwapim, Ga and the Suhum districts, respectively. Their sources may comprise either dolomite $(\mathrm{Ca} \mathrm{Mg}$ 
$\left.\left(\mathrm{CO}_{3}\right)_{2}\right)$ or calcite $\left(\mathrm{CaCO}_{3}\right)$ in the rock matrix, and, therefore, characterized by temporary hardness.

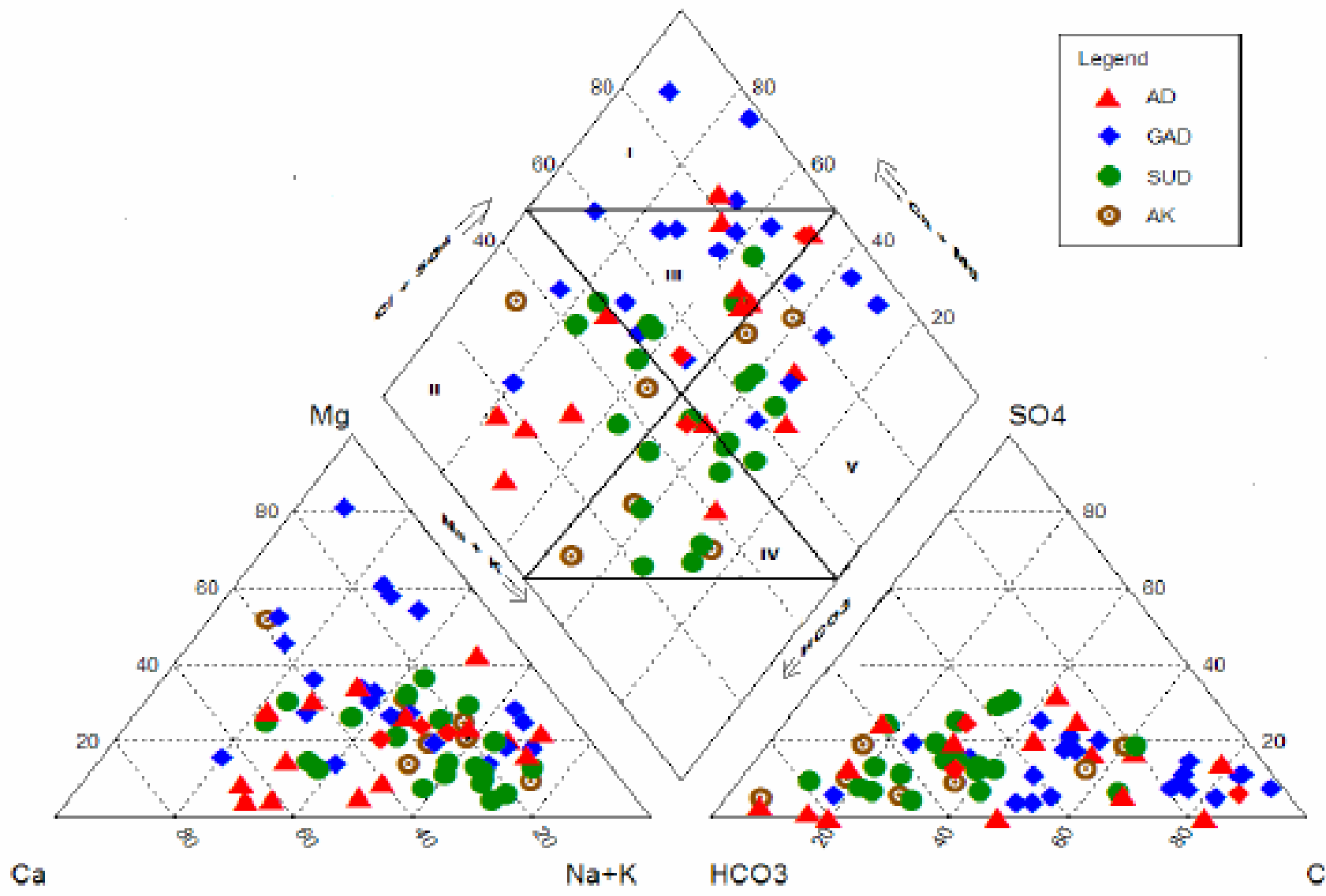

Fig. 8. Piper plot showing the chemical types of groundwater sources in the Densu basin

The $\mathrm{Na}-\mathrm{Cl}$ or $\mathrm{Na}-\mathrm{Cl}-\mathrm{HCO}_{3}-\mathrm{Cl}$ water type has saline characteristics. This water type occupies the section of the diamond shape designated as $\mathrm{V}$ and represents $22 \%$ of groundwater in the basin. This percentage is made up of 3\%, 3\%, 9\% and 7\% contributions from the East Akim, Akwapim, Ga and the Suhum districts, respectively. Their sources may comprise either of sea- or rain- water. Other water types that are of major importance in the explicit understanding of the water chemistry of groundwater in the Densu basin can be characterized as mixed waters. This include the $\mathrm{Na}-\mathrm{Mg}-\mathrm{Ca}-\mathrm{HCO}_{3}$ water. In this type, no particular cation predominates while $\mathrm{HCO}_{3}$ is the main anion. This water type occupies the section designated as IV and constitutes $21 \%$ of groundwater within the basin.

The $\mathrm{Na}^{+}-\mathrm{Cl}^{-}-\mathrm{SO}_{4}^{2-}$ water type has no particular cation which predominates, while $\mathrm{SO}_{4}^{2-}$ is the main anion. This water type occupies the section designated as III and forms $34 \%$ of groundwater within the basin. A minor water type, $\mathrm{Ca}-\mathrm{Mg}-\mathrm{SO}_{4}$ water (I field) of the diamond shape, have also been delineated within the basin. The source aquifers contain gypsum $\left(\mathrm{CaSO}_{4} \cdot 2 \mathrm{H}_{2} \mathrm{O}\right)$ or anhydrite $\left(\mathrm{CaSO}_{4}\right)$ that may have undergone dissolution and, therefore, characterized by permanent hardness. About $4 \%$ of ground-water within the basin consists of these aquifers. 
The major water types and $\mathrm{Na}^{+}-\mathrm{Cl}^{-}-\mathrm{SO}_{4}^{2-}$ water type depict rock-water interactions involving the dissolution of carbonates and rain- or sea-water by the recharging groundwater within the relatively permeable weathered zone above the underlying rocks. The rock types of the study area, as stated earlier, consist mainly of a complex of precambrian granite and granodiorite with associated gneiss underlying the larger part of the basin and the Togo series, which consists of sedimentary rocks and their metamorphosed versions (e.g. quartzite, schist, shale and phyllite). The rocks of the Togo series contain carbonates either in the form of impure limestone or as cement in the matrices of the sandstone (Kortatsi, 2005). These carbonates are the soluble type within the Togo series and, thus, constitute the source of $\mathrm{Ca}-\mathrm{Mg}-\mathrm{HCO}_{3}$.

\section{Conclusion}

The study showed that most of the chemical parameters in the waters of the Densu basin are within the WHO limits for drinking water, posing no health threat to consumers. However, the levels of nutrients, especially in the waters at Aponsahene $(105.8 \mathrm{mg} / \mathrm{l})$, Damang $(66.6 \mathrm{mg} / \mathrm{l})$, Mangoase (48.0 mg/l), Potrase (33.6 mg/l), Asuatwene (41.3 mg/l) and Afabeng (50.8 mg.1), and, possibly, all the other areas, where nitrate levels exceeded the critical value of $10 \mathrm{mg} / \mathrm{l}$, should be closely monitored. Human activities such as farming, washing and sewage disposal within the basin, perhaps, have great impact on the quality of groundwater. Groundwaters in the Ga and Suhum districts were found to be more mineralized, suggestive of heavy impact of human activities and existence of old waters.

Correlations between major ions showed expected process-based relationship between $\mathrm{Ca}^{2+}$ and $\mathrm{Cl}^{-}(r=0.86) ; \mathrm{Mg}^{2+}$ and $\mathrm{Cl}^{-}(r=0.84) ; \mathrm{Na}^{+}$and $\mathrm{SO}_{4}^{2-}(r=0.77) ; \mathrm{Na}^{+}$and $\mathrm{Cl}^{-}(r=0.75)$; $\mathrm{Mg}^{2+}$ and $\mathrm{SO}_{4}^{2-}(r=0.74) ; \mathrm{Mg}^{2+}$ and $\mathrm{Ca}^{2+}(r=0.71) ; \mathrm{Ca}^{2+}$ and $\mathrm{SO}_{4}^{2-}(r=0.58)$; and $\mathrm{K}^{+}$and $\mathrm{SO}_{4}{ }^{2-}(r$ $=0.51$ ), derived mainly from the geochemical and biochemical processes within the aquifer. Two major hydrochemical water types constituting about $41 \%$ of groundwater sources within the basin have been delineated. These are $\mathrm{Ca}-\mathrm{Mg}-\mathrm{HCO}_{3}$ water $(19 \%)$ and $\mathrm{Na}-\mathrm{Cl}$ or $\mathrm{Na}-\mathrm{Cl}-\mathrm{HCO}_{3}-\mathrm{Cl}$ water (22\%) types. Fifty-nine per cent of groundwater sources are mixed waters with no particular cation predominating, and having either $\mathrm{HCO}_{3}^{-}$or $\mathrm{SO}_{4}^{2-}$ ions as the main anion.

\section{Acknowledgement}

The study forms part of the comprehensive study of application of isotope techniques in groundwater assessment in the Densu basin by International Atomic Energy Agency (IAEA) and the Government of Ghana. Facilities provided are gratefully acknow-ledged. The authors also acknowledge the efforts of Ms Grace Dartey, a technician, of the Environmental Chemistry Division, CSIR-Water Research Institute, for assisting in the collection and analysis of samples, and Mr Harrison Komladjei, Principal Draughts-man, CSIR-Water Research Institute, for producing the map of the study area.

\section{References}

APHA (1998). Standard Methods for the Examination of Water and Wastewater, 20th edn. American Public Health Association, Washington, D.C

Appelo C. A. J. and Postma D. (1993). Geochemistry, groundwater and pollution. Balkema, Rotterdam.

Baird C. (1995). Environmental chemistry, 2nd edn. W.H. Frreemen and Co., New York.

Barcelona M., Gibb J. P. Helfrich J. A. and Garske E. E. (1985). Practical guide for groundwater sampling. Ilinois State Water Survey ISWS Contract Report 37.

Bockris J. O. M. (1978). Environmental chemistry. Premium Press, New York. 332 pp.

Bush D. and Mayer M. (1982). A case of infantile methemoglobinemia in South Dakota. J. Envir. Hlth 44 (6): 310 311.

Comly H. H. (1945). Cyanosis in infants by nitrates in well water. Am. Med. Ass. J. 129 (2): 112-116.

Claasen H. C. (1982). Guidelines and techniques for obtaining water samples that accurately represent the quality of an aquifer. U.S. Geological Survey Open File Report 82-1024. 49 pp.

West African Journal of Applied Ecology, Vol. 12, 2008 
Cotruvo J. A (1988). Drinking water standards and risk assessment. Regul. Toxic. Pharmac. 8(3): 288.

Dalas H. F. and Day J. A. (1993). The Effect of Water Quality Variables on River and Ecosystem. Water Research Commission Report No TT 61/93.

Dickson K. B. and Benneh G. (1980). A New Geography of Ghana. Longman, London.

Daily Graphic (2001). Ghana Newspaper, September 14. Farming along Densu banned, a report by Boahene Asamoah and Ceasar Abagali. p. 32.

Daily Graphic (2001). Ghana Newspaper, September 18, Use water resources judiciously- Bartels, a report by Ceasar Abagali and Samuel Ato Afful. p. 16.

Evans D. W., Cutshall N. H., Cross F. H. and Wolfe D.A. (1977). Manganese cycling in the Newport estuary, North Carlifonia. Estuar. Cstl Mar. Sci. 5: 71-80.

Junner N. R. (1940). Geology of the Gold Coast and Western Togoland with revised Geological map: Gold Cst Geol. Surv. Bull. 11 40-49.

Kempster P. L., Van Vliet H. R. and Kuhn A. (1997). The need for guidelines to bridge the gap between ideal drinking-water quality and that quality which is practically available and acceptable. Water SA 23(2) 163-167.

Karanth K. R. (1994). Groundwater assessment development and management, 3rd reprint. Tata McGraw-Hill Publishing Companay Limited, New Delhi.

Kelter P. B., Grundman J., Hage D. S. and Carr J. D. (1997). A discussion of water pollution in the US and Mexico with high school laboratory activities of lead, anthrazine and nitrate. Chem. Educ. 74 (12): 1413-1418.

Kortatsi B. K. (2005). Geochemical assessment of springs in the Ho District of Ghana using multivariate statistical technique. West Afr. J. appl. Ecol. 7: 83-94.

Ministry of Health (1999). Medium term Health Strategy towards Vision 2020. Ministry of Health, Accra, Ghana.

Piper A. M. (1994). Agraphic procedure in the Geochemical interpretation of water analyses. Am. Geophys. Un. Trans. 25: 914-923.

Stumn W. and Morgan J. J. (1981): Aquatic Chemistry. Willey, New York. 780 pp.

WHO (1993). Guidelines for Drinking Water Quality. World Healthy Organization, Genava, Switzerland.

WHO (1996). Guideline for Drinking Water Quality, 2nd edn. WHO, Geneva. pp. 351-354. 